\title{
Possibilities and benefits of intermediate care units in healthcare systems from a logistics perspective
}

\author{
Aili Biriita Bertnum ${ }^{[0000-0003-3092-5760]}$, Giuseppe Ismael Fragapane ${ }^{[0000-0001-8915-509 X]}$, \\ Marco Semini and Jan Ola Strandhagen ${ }^{[0000-0003-3741-9000]}$ \\ Department of Mechanical and Industrial Engineering, Norwegian University of Science and \\ Technology, 7491 Trondheim, Norway \\ \{aili.b.bertnum, giuseppe.fragapane, marco.semini, ola.strandhagen\} \\ antnu.no
}

\begin{abstract}
Intermediate care units have been established as a response to the emerging challenges of healthcare systems to maintain high quality and continuous care. While the term is well known in both literature and practice, it lacks a unified definition. There is no common consensus of how intermediate care units can be successfully implemented and properly utilized in healthcare systems. Large variations of services in intermediate care units can be found. This literature review has structured the existing research on intermediate care units, identifying the possibilities and benefits of intermediate care units in healthcare systems from a logistics perspective. The main findings discussed in this study concern the following topics: the effect of intermediate care units on healthcare system performance and patient outcomes, and potential users of and services provided by intermediate care units. This study presents the state-of-the-art research on intermediate care units and suggests topics for further research.
\end{abstract}

Keywords: Intermediate Care Units, Healthcare System, Logistics.

\section{Introduction}

A high quality and well-performing healthcare system is essential for the health and welfare of the population. An ongoing trend is to shift from expensive inpatient treatments to more cost-efficient outpatient treatments. This is done by investing in primary care making it responsible for coordination of care, while reducing the number of hospital beds and length of hospital stays [1]. However, investments in primary care is not following the same pace as the scale-down of inpatient care, making coordination of healthcare services a challenge.

Poorly coordinated and fragmented care in interfaces of the healthcare system can result in unmet needs of complex health $[1,2]$. Elderly are known to have complicated care pathways. Statistics show that $89 \%$ of people aged 85 and above have at least two chronic conditions [1]. In addition, the population of elderly is ever-increasing, from $9 \%$ in 1960 to $17 \%$ in 2015 on average for the population of OECD countries [2]. An ageing population increases the demand for long-term care [2, 3] and respectively the 
pressure on the healthcare system. Expenditures for long-term care are also increasing more compared to other healthcare services [2].

Intermediate care units are established to support the healthcare systems in facing the challenges of coordination and long-term care and involves healthcare provided in the interfaces of the healthcare system $[4,5]$. The term, intermediate care, is widely used in both literature and practice, but there is no unified definition of the term $[4,6$, 7]. E.g., it is used both for a unit in a hospital and for a separate facility between primary and secondary care [8]. There are widespread effects of intermediate care units found in literature and few attempts of comparisons for generalization. Implying a need for comparing different intermediate care units [7].

There are opportunities within the field of logistics to support the challenges of the healthcare system. It can assist healthcare workers and act as the foundation for building healthcare operations [9]. This study will identify and structure the existing research on intermediate care units, with the aim of showing the possibilities and benefits of intermediate care units in healthcare systems. In addition, the study will suggest topics for further research.

\section{Background}

In most western countries, the healthcare system consists of two main sectors. The primary sector provides preventive healthcare, diagnostics and treatment, medical rehabilitation, nursing services and emergency care [2]. The general practitioner at the primary sector is often the first point of contact in the healthcare system $[2,10]$. The primary sector aims at fulfilling the basic and emergent needs of the patients, and is thus a decentralized service provider closely located to the patients.

The patient is referred to the secondary sector by the general practitioner, but in some countries the patient can choose to approach secondary sector directly [2]. The secondary sector consists of hospitals and specialist healthcare institutions [11], and is responsible for operation of inpatient and outpatient clinics, and ambulance and laboratory services. The secondary sector is a centralized service provider aiming at providing specialized care. Due to comprising expensive and limited resources [12], cost- and resource-efficient operation of hospitals is desirable. After hospital discharge the general practitioner is responsible for the medical follow-up of the patient [11].

Logistics involves both internal and external healthcare operations [9]. Logistics can be defined as the strategic management of procurement, movement and storage of materials and inventory for maximized profitability through cost-effective order fulfilment [13]. Management of logistical activities relates to the planning and coordination of the activities required for providing the demanded service at the highest quality and lowest cost possible [13].

Being concerned with providing high-quality healthcare services to the needs of the patients, the healthcare system can be characterized as a service supply chain. The demand of healthcare services is expressed by patient flow [12]. A strategic fit between the supply chain strategy and the competitive strategy is achieved by having knowledge of both the uncertainties related to customers and the supply chain, and the capabilities 
of the supply chain [14]. Having strategic fit involves providing the desired level of responsiveness at the lowest possible cost [14].

The healthcare system should be designed like production systems, with the aim of minimizing waiting times between referral and service provision, synchronizing between utilization of healthcare resources and patient flow, and identifying and fixing bottlenecks in the healthcare system [10].

\section{Research design}

The research was performed in two steps. First, an initial literature search was performed to increase the knowledge of intermediate care units. It identified keywords related to the most common terms, aims and outcomes of intermediate care units, as shown in Table 1. Second, a literature review was performed based on the keywords to get an overview of the existing research on intermediate care units. Literature reviews are often used to identify, evaluate and synthesize the existing body of knowledge [15].

Several databases (Web of Science, Scopus and Google Scholar) were selected to get a more reliable body of knowledge. The keywords and relevant synonyms were combined in the literature review. Table 1 illustrates the use of the common Booleans "AND" and "OR". In addition, the Booleans "*” and "?" were used for including different endings and different spellings of the word.

Table 1. Keywords used in the literature review.

\begin{tabular}{|c|c|c|c|c|}
\hline Terms & & Aims & & Outcomes \\
\hline $\begin{array}{c}\text { Intermediate care } \\
\text { OR } \\
\text { Community hospital } \\
\text { OR } \\
\text { Nursing home }\end{array}$ & $A N D$ & $\begin{array}{c}\text { Coordination } \\
\text { OR } \\
\text { Cooperation } \\
\text { OR } \\
\text { Integration } \\
\text { OR } \\
\text { Continuity } \\
\end{array}$ & $A N D$ & $\begin{array}{c}\text { Admission } \\
O R \\
\text { Length of stay } \\
O R \\
\text { Readmission } \\
O R \\
\text { Discharge }\end{array}$ \\
\hline
\end{tabular}

The publications were chosen if the title and abstract indicated that the research topic would concern the definition, utilization or effect of intermediate care on healthcare systems. Based on the rest of the publication, it was excluded if the main objective was to discuss medical or mental illnesses instead of intermediate care. In addition, publications from references and relevant publications from the initial literature search were included. The literature review identified 19 publications as relevant for this study. 


\section{$4 \quad$ Results}

The identified research in the literature review provided several results. The geographical distribution of publications is shown in Table 2. The publications are structured by publication year in Table 3 . In Table 4 the suggested outcomes and potential utilization areas of intermediate care units are divided into effect on the overall healthcare system and on patients receiving treatment, potential users of intermediate care units, and potential services provided by intermediate care units.

Table 2. Geographical distribution of publications.

\begin{tabular}{lcl}
\hline Country & Publications & References \\
\hline United Kingdom & 12 & {$[5,16-26]$} \\
Unites States & 3 & {$[27-29]$} \\
Netherlands & 2 & {$[6,8]$} \\
Norway & 1 & {$[3]$} \\
Italia & 1 & {$[30]$} \\
\hline
\end{tabular}

Table 3. Number of publications by year.

\begin{tabular}{lcl}
\hline Publication year & Publications & References \\
\hline Before 2000 & 1 & {$[26]$} \\
$2001-2005$ & 4 & {$[5,21-23]$} \\
$2006-2010$ & 5 & {$[16,25,27-29]$} \\
$2011-2015$ & 7 & {$[3,6,18-20,24,30]$} \\
$2016-2017$ & 2 & {$[8,17]$} \\
\hline
\end{tabular}

Table 4. Outcomes and utilizations of intermediate care units

\begin{tabular}{lcl}
\hline Effect on patient and healthcare system & Publications & References \\
\hline Reduction of unnecessary hospital admissions & 9 & {$[3,6,18,20-24,27]$} \\
Reduction of prolonged hospital stays and de- & 9 & {$[3,5,6,16,20-24]$} \\
layed discharges from hospitals & 3 & {$[3,23,28]$} \\
Support independent living & 2 & {$[6,23]$} \\
Reduction of long-term admissions & 2 & {$[3,17]$} \\
Continuity of care & 2 & {$[18,23]$} \\
Reduction of travel distance & & \\
\hline Identified patient types & 8 & {$[3,5,21,24,25,28-30]$} \\
\hline Elderly & 2 & {$[26,29]$} \\
Patients with chronic diseases & 1 & {$[8]$} \\
Surgical patients & 1 & {$[30]$} \\
Disabled patients & & \\
\hline Identified healthcare services & 5 & {$[17,21,22,26,30]$} \\
\hline Rehabilitation and therapeutic care & 3 & {$[27,29,30]$} \\
Hospice care & 2 & {$[5,24]$} \\
Geriatric care & 2 & {$[16,24]$} \\
Social care & 2 & {$[24,26]$} \\
Acute care & 2 & {$[26,29]$} \\
Palliative care & &
\end{tabular}




\section{Discussion}

Intermediate care is receiving more attention than before. Table 3 illustrates an increasing number of publications from 1993 to 2017 indicating that it is a topic of current and increasing interest. Research on intermediate care units is mostly happening in Europe, being the origin of $85 \%$ of publications in the literature review, as shown in Table 2 . United Kingdom seems to be the foremost driver of research on and establishment of intermediate care with nearly $2 / 3$ of the publications. In addition, $86 \%$ of localities in England are approaching the integration of or have integrated intermediate care units [16]. United States is the second largest contributor to research on intermediate care, as shown in Table 2. The literature review implies that intermediate care is not a highly researched topic in eastern countries as publications only originate from Europe and the United States.

Intermediate care units are centered on the needs of the patient and can improve the overall quality of healthcare system performance. Patients generally have positive perceptions of intermediate care [19]. Research also shows that intermediate care can result in improved outcomes for all patient types $[17,18]$. E.g., providing hospice services in intermediate care units has shown to be beneficial for both hospice and non-hospice patients [27].

The literature review implies that elderly are considered the main user of intermediate care, as shown in Table 4. Elderly constitute an ever-increasing part of the population, and increase the pressure on the healthcare system requiring a larger amount of customized services compared to the rest of the population. With the pressures on costand resource-efficiency in healthcare systems [12], it is natural to focus on the most resource-needing patient type. However, it does not demonstrate that elderly are the only patient type suitable for intermediate care. It can imply that the knowledge and experience required to treat complex patient needs provides flexibility to treat many different patient types.

Large variations are found in which services that are or should be provided in intermediate care units $[6,7,16,19]$. The literature review shows similar findings, as summarized in Table 4. There are concerns that intermediate care unit is a top-down solution that is poorly suited to real-life situations [31], which is strengthened with the fact that intermediate care units do not seem to have a standardized structure. Instead of accommodating to an ideal-model or choose between a set of predefined models, intermediate care units are implemented with the healthcare services that fit the local healthcare system and patient needs [22].

The customization of intermediate care units to local needs can be both an advantage and a disadvantage. Without a standardized structure, or at least some predefined guidelines, it is difficult to measure performance and success of implementation. In worst case, the diversity of intermediate care units can imply a poor adherence to existing models resulting in poor clinical outcomes and inefficiency [22]. However, by complying with local needs, intermediate care units are better equipped to handle the uncertainties and large variations in demand associated with the healthcare environment. Each patient is unique regarding the combination of age and health. 
Intermediate care units show possibilities and benefits for both patients and the healthcare system. Intermediate care units can reduce waiting times by having the responsiveness to handle uncertain demand; can contribute to improved synchronization between healthcare resources and patient flow by having the flexibility to treat many types of patients; and can fix bottlenecks by adapting to local needs and provide customized services. It can be characterized as a collection of healthcare services complementing the existing healthcare system [22]. The integration with primary and secondary sector is of importance $[17,19,24]$. There is a need to map and characterize different healthcare environments to be able to match and successfully implement intermediate care units. It involves identifying and defining a set of intermediate care units, where each type is suitable for a specific healthcare system. It will require greater availability of comparable data on cost, service intensity and quality of care [27].

\section{Conclusion}

This study has identified and structured the existing research on intermediate care units. A current and increasing research interest of intermediate care units in Europe and the United States has been highlighted. In addition, the study has identified possibilities and benefits for healthcare systems with intermediate care units and for patients receiving treatment at intermediate care units.

The effects of intermediate care units on healthcare systems and patient flow are the following. Intermediate care units can reduce waiting times through responsiveness to handle uncertain demand; can improve synchronization between healthcare resources and patient flow through flexibility to treat many types of patients; and can reduce bottlenecks through providing customized services to local healthcare needs. There is still a need to identify and define a set of intermediate care units suitable for different healthcare systems.

The limitations of this study are related to the difficulties of identifying the full body of knowledge, as there is no common understanding of intermediate care units. The literature review of 19 papers is able to show research trends, but not the full picture of the current situation.

Future research should focus on mapping different healthcare systems and identify the proper utilization and service provision of intermediate care units in each case, in terms of identifying the desired level of responsiveness at the lowest possible cost. In addition, investigating the emergence of intermediate care units in eastern countries can also be a topic of interest, as it was not represented in this study.

Acknowledgement. This research received funding from the strategic research area NTNU Health in 2018 at NTNU, Norwegian University of Science and Technology. The authors also want to gratefully acknowledge the structured literature review performed by Nathalie Bruntveit Madsen and Ida Borgen Vaule, master students at NTNU, which provided the initial structure of this study. 


\section{References}

1. Berchet, C. and Forde, I., Caring for quality in health: Lessons learnt from 15 reviews of health care quality. OECD Publishing (2017).

2. OECD, Health at a Glance 2017: OECD Indicators. OECD Publishing, Paris (2017).

3. Dahl, U., Steinsbekk, A., Jenssen, S., and Johnsen, R., Hospital discharge of elderly patients to primary health care, with and without an intermediate care hospital-a qualitative study of health professionals' experiences. International Journal of Integrated Care 14(2), 1-11 (2014).

4. Melis, R.J.F., Rikkert, M.G.M.O., Parker, S.G., and van Eijken, M.I.J., What is intermediate care? An international consensus on what constitutes intermediate care is needed. British Medical Journal 329(7462), 360-361 (2004).

5. Steiner, A., Intermediate care - a good thing? Age and Ageing 30, 33-39 (2001).

6. Millar, A.N., Hughes, C.M., Passmore, A.P., and Ryan, C., Intermediate Care: The Role of Medicines Management. Drugs \& Aging 31(1), 21-31 (2014).

7. Prin, M. and Wunsch, H., The Role of Stepdown Beds in Hospital Care. American Journal of Respiratory and Critical Care Medicine 190(11), 1210-1216 (2014).

8. Plate, J.D.J., Leenen, L.P.H., Houwert, M., and Hietbrink, F., Utilisation of Intermediate Care Units: A Systematic Review. Critical Care Research and Practice 2017, 1-10 (2017).

9. Kros, J.F. and Brown, E.C., Health Care Operations and Supply Chain Strategy, in Health Care Operations and Supply Chain Management: Strategy, Operations, Planning, and Control. John Wiley \& Sons, Incorporated, Somerset (2012).

10. Hall, R., Belson, D., Murali, P., and Dessouky, M., Modeling Patient Flows Through the Health care System, in Patient Flow: Reducing Delay in Healthcare Delivery. Springer US, Boston, 3-42 (2013).

11. Røsstad, T., Garåsen, H., Steinsbekk, A., Sletvold, O., and Grimsmo, A., Development of a patient-centred care pathway across healthcare providers: a qualitative study. BMC Health Services Research 13(1), 121 (2013).

12. Côté, M.J., Understanding Patient Flow. Decision Line 31(2), 8-10 (2000).

13. Christopher, M., Logistics \& Supply Chain Management. Pearson Education, Edinburgh, United Kingdom (2016).

14. Chopra, S. and Meindl, P., Supply Chain Management: Strategy, Planning, and Operation. Sixth edition ed. Pearson Education Limited Harlow, England (2016).

15. Okoli, C. and Schabram, K., A guide to conducting a systematic literature review of information systems research. (2010).

16. Martin, G.P., Hewitt, G.J., Faulkner, T.A., and Parker, H., The organisation, form and function of intermediate care services and systems in England: results from a national survey. Health and Social Care in the Community 15(2), 146-154 (2007).

17. Cornes, M., Whiteford, M., Manthorpe, J., Neale, J., Byng, R., Hewett, N., Clark, M., Kilmister, A., Fuller, J., Aldridge, R., and Tinelli, M., Improving hospital discharge arrangement for people who are homeless: A realist synthesis of the intermediate care literature. Health and Social Care in the Community 26, e345-e359 (2017).

18. Pearson, M., Hunt, H., Cooper, C., Shepperd, S., Pawson, R., and Anderson, R., Providing effective and preferred care closer to home: a realist review of intermediate care. Health and Social Care in the Community 23(6), 577-593 (2015). 
19. Young, J., Gladman, J.R.F., Forsyth, D.R., and Holditch, C., The second national audit of intermediate care. Age and Ageing 44, 182-184 (2015).

20. Szczepura, A., Residential and nursing homes: how can they meet the challenge of an aging population? Aging Health 7(6), 877-887 (2011).

21. Nancarrow, S., Improving Intermediate Care: Giving Practitioners a Voice. Journal of Integrated Care 12(1), 33-41 (2004).

22. Martin, G.P., Peet, S.M., Hewitt, G.J., and Parker, H., Diversity in intermediate care. Health \& social care in the community 12(2), 150-154 (2004).

23. Roe, B. and Beech, R., Intermediate and Continuing Care: Policy and Practice. Blackwell Publishing Ltd, Oxford, United Kingdom (2005).

24. Philp, I., Mills, K.A., Thanvi, B., Ghosh, K., and Long, J.F., Reducing hospital bed use by frail older people: results from a systematic review of the literature. International Journal of Integrated Care 13, 1-19 (2013).

25. Young, J., The development of intermediate care services in England. Archives of Gerontology and Geriatrics 49(Suppl. 2), S21-S25 (2009).

26. McCormack, B., The developing role of community hospitals: an essential part of a quality service. Quality in Health Care 2(4), 253-258 (1993).

27. Stevenson, D.G. and Bramson, J.S., Hospice Care in the Nursing Home Setting: A Review of the Literature. Journal of Pain and Symptom Management 38(3), 440-451 (2009).

28. Murray, L.M. and Laditka, S.B., Care Transition by Older Adults From Nursing Homes to Hospitals: Implications for Long-Term Care Practice, Geriatrics Education, and Research. Journal of the American Medical Directors Association 11, 231-238 (2010).

29. Hanson, L.C. and Ersek, M., Meeting Palliative Care Needs in Post-Acute Care Settings. Journal of American Medical Association 295(6), 681-686 (2006).

30. Longo, F., Salvatore, D., and Tasselli, S., The growth and composition of primary and community-based care services. Metrics and evidence from the Italian National Health Service. BMC Health Services Research 12(393), 1-13 (2012).

31. Evans, J.G. and Tallis, R.C., A new beginning for care for elderly people? Not if the psychopathology of this national service framework gets in the way. British Medical Journal 322(7290), 807-808 (2001). 